\title{
Application of High-Throughput Seebeck Microprobe Measurements on Thermoelectric Half-Heusler Thin Film Combinatorial Material Libraries
}

\author{
Pawel Ziolkowski ${ }^{\mathrm{a}^{*}}$, Matthias Wambach ${ }^{\mathrm{b}}$, Alfred Ludwig $^{\mathrm{b}}$, Eckhard Mueller $^{\mathrm{a}, \mathrm{c}}$ \\ ${ }^{a}$ Institute of Materials Research, German Aerospace Center, D-51170 Cologne, Germany \\ ${ }^{\mathrm{b}}$ Institute for Materials, Ruhr-University Bochum, D-44801Bochum, Germany \\ ${ }^{\mathrm{c}}$ Institute for Inorganic and Analytical Chemistry, Justus Liebig University Giessen, \\ Heinrich-Buff-Ring 17, D-35392 Giessen, Germany
}

\begin{abstract}
In view of the variety and complexity of thermoelectric (TE) material systems, combinatorial approaches to materials development come to the fore for identifying new promising compounds. The success of this approach is related to the availability and reliability of high-throughput characterization methods for identifying interrelations between materials structures and properties within the composition spread libraries. A meaningful characterization starts with determination of the Seebeck coefficient as a major feature of TE materials. Its measurement, and hence the accuracy and detectability of promising material compositions, may be strongly affected by thermal and electrical measurement conditions. This work illustrates the interrelated effects of the substrate material, the layer thickness, and spatial property distributions of thin film composition spread libraries, which are studied experimentally by local thermopower scans by means of the Potential \& Seebeck Microprobe (PSM). The study is complemented by numerical evaluation. Material libraries of the half-Heusler compound system Ti-Ni-Sn were deposited on selected substrates (Si, AlN, $\mathrm{Al}_{2} \mathrm{O}_{3}$ ) by magnetron sputtering. Assuming homogeneous properties of a film, significant decrease of the detected thermopower $S_{\mathrm{m}}$ can be expected on substrates with higher thermal conductivity, yielding an underestimation of materials thermopower between $15 \%$ and 50\%, according to FEM simulations. Thermally poor conducting substrates provide a better accuracy with thermopower underestimates lower than $8 \%$, but suffer from a lower spatial resolution. According to FEM simulations, local scanning of sharp thermopower peaks on lowly conductive substrates is linked to an additional deviation of the measured thermopower of up to $70 \%$ compared to homogenous films, which is $66 \%$ higher than for corresponding cases on substrates with higher thermal conductivity of this study.
\end{abstract}

Keywords: Seebeck coefficient, thermoelectric, thin films, Potential \& Seebeck Microprobe, combinatorial material development, high-throughput characterization, half-Heusler compounds, measurement accuracy, spatial resolution

\section{Supplementary Information}

- Spatial maps of the element concentration, the Seebeck coefficient, the resistivity, and film thickness of all characterized material libraries in comparison for each substrate material.

* Corresponding author. Tel.: +49-(0)2203-6013576, E-mail address: pawel.ziolkowski@dlr.de 
- Diffractograms from microstructure characterization of the films on different substrates using X-Ray Diffraction.

\section{Introduction}

Decreasing fossil fuel supplies and rising greenhouse emissions increase the need for sustainable energy sources and efficient energy use. Conversion of waste heat to electric power by thermoelectric (TE) devices [1] is considered to improve the energy efficiency of combustion engines and to harvest energy from sunlight or nuclear decay. TE materials are typically designed for specific operation temperatures, ranging from far below ambient $(100 \mathrm{~K}[2,3])$ to very high temperatures (e.g. $1300 \mathrm{~K}$ [4]). The conversion efficiency is determined by the dimensionless figure of merit $z T\left(z T=S^{2} \sigma \kappa^{-1} T, S-\right.$ Seebeck coefficient / thermopower, $\kappa$ - thermal conductivity, $\sigma-$ electrical conductivity, $T$ - absolute temperature). The figure of merit can be optimized by maximizing the power factor $S^{2} \sigma$, while the thermal conductivity must be minimized. The properties are interdependent by relations from solid state physics but can be shifted by composition, doping, microstructural effects and nanostructuring. In this context, increasing chemical complexity [5,6] and nanostructuring [7-13] are discussed to reduce the lattice contribution to the thermal conductivity by increased phonon scattering [14], which can improve $z T$ values due to different transfer characteristics for phonons and charge carriers.

Among thermoelectric materials, half-Heusler (HH) compounds are promising, with high conversion efficiency for both $\mathrm{p}$ - and n-type conductivity. They exhibit semiconducting characteristics although formed from metallic elements only. They mainly consist of non-critical chemical constituents and give broad access to materials optimization by means of nanostructuring and optimization of the charge carrier concentration by doping or self-doping in off-stoichiometric samples [15]. Although research on these compounds is ongoing since decades [16,17], a high conversion efficiency with a $z T$ close to 1.5 has been achieved only recently. High material performance has initially been demonstrated in the $500-900 \mathrm{~K}$ temperature range for the optimized multinary composition $\left(\mathrm{Zr}_{0.5} \mathrm{Hf}_{0.5}\right)_{0.5} \mathrm{Ti}_{0.5} \mathrm{NiSn}_{0.998} \mathrm{Sb}_{0.002}$ [18] and has triggered further research activities where outstanding conversion efficiencies were reproduced by precise control of the nanostructure [19-21]. A variety of possible synthesis routes, chemical compositions and multiple means for efficiency enhancement make $\mathrm{HH}$ compounds an ideal candidate for combinatorial materials research.

Rapid fabrication and high-throughput characterization of large numbers of samples with distinct compositions is one advantage of combinatorial materials science. It allows for efficient performance assessment and can reveal structure-property relationships for a desired compositional range within a material system [22-27].

High sample throughput at the highest possible quality is one of the major features for applied characterization techniques in combinatorial material development. Concerning the determination of transport properties, sound probing methods come along as most suitable to fulfill these generally conflicting requirements, since they have been deeply investigated methodically in many cases and offer high time-saving potential, especially in consideration of simultaneous methods, capable to determine multiple physical properties in one experiment.

The challenge of locally measuring thermal properties [28-30] is an ultimate precondition for a local $z T$ mapping on a materials library (ML) that has not been fully accomplished yet. However, a 
meaningful characterization of TE performance is already achieved by the determination of the power factor. The constituent material properties $(S, \sigma)$ must fit within certain ranges for high potential materials, e.g. in terms of their dependence on the charge carrier concentration [4]. Consequently, the determination of the spatially resolved power factor by measuring the electrical conductivity and the Seebeck coefficient is a cornerstone for efficient assessment of TE materials performance.

For rapid and precise determination of the electrical conductivity contact-based methods as 4-point [31-34] or van der Pauw setups [35,36] are state of the art. Related to Si-based microsystem technologies probing methods for the electrical conductivity became mandatory for the development of semiconducting and insulating materials for the use in device structures of diodes and transistors. Metrological devices consequently reached commercial relevance and are available in a variety, such as stand-alone-instruments [37] or accessory equipment for scanning electron microscopy (SEM) and AFM-based cantilevers [38,39]. For high-throughput characterization of ML customized test stands have been developed for mapping electrical properties both at room temperature and elevated temperatures, which have been used, e.g., for identifying functional materials which show reversible phase transformations [40].

For the measurement of the local Seebeck coefficient a high diversity of predominantly custom-built methods are applied since TE device concepts reached a by far lower presence in the market than silicon technologies. In principle, the compositional gradient and the required local resolution of the characterization methods are interlinked to each other. Both determine the achievable sample throughput and the measurement density and accuracy of the transport properties correlated to compositional or structural variations of the studied compounds. Although many specific measurement setups have been described, less attention was paid to inherent sources of uncertainties, which possibly arise from particular constructive and metrological solutions and may differ in their impact on the measured thermopower depending on whether bulk or thin film samples are studied.

In principle, the reported probing techniques for the measurement of the Seebeck coefficient have similar methodical approaches. A local temperature gradient is established within a sample by a heated probe tip. The applied temperature difference is recorded together with the thermovoltage. The temperature difference and the thermovoltage must be measured exactly at the same place, which usually is done by the readings of two different thermocouples, each connected to the probe and sample respectively. Itaka et al. [41] described a pin-probe type multi-channel measurement system which allows measuring Seebeck coefficient and electrical resistivity simultaneously for 10 samples in 15 minutes on a thin-film composition spread (TFCS) library, at different base temperatures between 10 and $400 \mathrm{~K}$. Otani et al. [42] described a four-point method with spring-loaded probe tips, capable of measuring the Seebeck coefficient and the electrical conductivity within $20 \mathrm{~s}$ for each measurement area. Funahashi et al. [43] published a Seebeck tester for sol-gel derived ML, composed of two thermocouples and heating elements capable of measuring 10 samples per minute. Generally, probing setups using a pattern of commercially available spring-loaded probe tips possess the disadvantage of a coarse probing pitch since they can be mounted with a minimum distance of approximately $100 \mu \mathrm{m}$ [44]. Other difficulties arise when standard sheathed thermocouples are utilized as single probing elements [45-47] because of their comparatively wide contacting area due to a minimum radius of curvature not lower than approximately $100 \mu \mathrm{m}$. This not only limits the probing pitch in the scanning mode but also the 
accuracy of the Seebeck measurement. Due to the geometrical distance between the temperature sensing point inside the thermocouple and the electrical contact point to the sample, the heat flow, which generally is flowing through the probing tip into or out of the sample, will establish a small temperature difference between the sensing point and the sample temperature at the electrical contact point. This effect is known from integral measurement methods as the cold finger effect $[48,49]$, and yields a significant underestimation of thermopower in scanning techniques as well. Practically, this error scales inversely proportional to the thermal conductance of the used probing tip and becomes larger as the distance (and with that thermal resistance) between sensor point and contact point increases, e.g. if thermally poor conducting sheath materials of thermocouples are used as electrical probes to the sample. Furthermore, the measured thermovoltage at a certain probe position is affected by adjacent regions of different Seebeck coefficient and the spatial distribution of the thermal and electrical conductivity within the radius of the spread of the temperature gradient around the contact area. This might cause problems when imaging sharp property transitions [51]. By means of adapted probing tips and fast data acquisition it is feasible to increase both the spatial resolution and the absolute accuracy of captured Seebeck coefficients to a certain extent [50-52].

However, only few investigations on the accuracy and resolution of probing methods for the determination of the local thermopower $S$ have been reported so far, mainly against the background of bulk sample measurements and generally not in the context of combinatorial TFCS material type libraries. Thus, the focus of this work is to give a review of methodical constraints and difficulties that arise during local thermopower characterization on compositionally graded film samples. As thin film samples represent the major route for combinatorial material science to date, numerical investigations on the Potential \& Seebeck Microprobe [53] are accompanied in this work by experimental data, obtained on sputter-deposited Ti-Ni-Sn based TFCS HH libraries [15]. Special attention is paid to the substrate material and the sample configuration, as both show a distinct impact on the accuracy and resolution of local measurements of the Seebeck coefficient. Data from transport property and chemical analyses obtained on $\mathrm{HH}$ library deposited on a Si wafer is reproduced from a previous work [15] and extended by additional results obtained on different substrates in this work.

\section{Experimental}

\section{Preparation of half-Heusler thin film composition spread material libraries}

The combinatorial libraries presented in this paper were fabricated by a combinatorial sputter method on lithographically structured substrates, see Fig. 1. The three MLs discussed in this paper were fabricated in one process by placing three substrates on a turn-table substrate holder and depositing material from elemental sources onto them. By rotating the substrate holder, each substrate gets exposed to the same deposition flux from the cathodes per revelation. A composition gradient at each cathode is realized by static apertures mounted in front of the cathode. By rotating the substrate holder in the range of $1 \cdot 10^{3}$ times, films with a thickness in the $10^{2} \mathrm{~nm}$-range are grown. Details of the process and the used sputter system can be found in [15]. The pattern divides the substrate into square measurement areas that are confined by crosses in all four corners. The center of the measurement areas is the reference point for the characterization of the properties of each individual measurement area. The centers of adjacent measurement areas have a distance of $4.5 \mathrm{~mm}$.

On the rotating main table, three different $100 \mathrm{~mm}$ diameter substrates were mounted: a single crystalline $\mathrm{Si}$ wafer with $1.5 \mu \mathrm{m}$ thermal $\mathrm{SiO}_{2}$, a polycrystalline $\mathrm{Al}_{2} \mathrm{O}_{3}$ and a polycrystalline $\mathrm{AlN}$ 
wafer. Each library contains a continuously graded composition distributed over the entire wafer surface. The maximum mean surface roughness Ra of the substrates was approximately $<1 \mathrm{~nm}$ for the $\mathrm{SiO}_{2} / \mathrm{Si}$ and $\sim 20 \mathrm{~nm}$ for the $\mathrm{Al}_{2} \mathrm{O}_{3}$ and $\mathrm{AlN}$ substrates. The sputter system allows for simultaneous fabrication of the three MLs in a single processing step by mounting multiple sample holders on the rotating main table. This results in near identical composition spreads on all three MLs. All MLs were annealed ex-situ in vacuum at $873 \mathrm{~K}$ for 4 hours.

\section{High-throughput measurement of chemical composition and electrical conductivity}

The composition of the ML was characterized by energy-dispersive X-ray analysis (EDX) using an EDX detector (Inca X-Act, Oxford Instruments plc) attached to a scanning electron microscope (SEM, Jeol 5800 SEM, Jeol Ltd.). The results are shown in Fig. 2. The excited area in the center of each measurement area is around $250 \mu \mathrm{m} \times 200 \mu \mathrm{m}$ and the error in the chemical composition is estimated to be \pm 1 at.\%. The electrical resistivity was calculated by combining data from measuring the sheet resistance of the libraries using an in-house-made 4-point probe setup [40] and the film thickness measured by profilometry (XP2, Ambios Technology), see Fig. 3 and 4. The 4-point probe pins have a distance of $500 \mu \mathrm{m}$. The thickness of the film at the center of the measurement area is calculated by averaging the thickness of the film at the crosses in all four corners. The typical film thickness in the center of the ML is set to $500 \mathrm{~nm}$.

High-throughput Seebeck characterization of combinatorial thin film samples by means of the Potential \& Seebeck Microprobe (PSM)

Seebeck coefficient measurements on the ML were made using the PSM [53]. This scanning setup consists of a heated probe tip, a three axes positioning stage, two type $\mathrm{T}$ thermocouples, and the data acquisition system. The base body of the tip is made of electrolytic $\mathrm{Cu}$ and carries an enameled resistive heating wire. A tungsten carbide (WC) tip with a radius of curvature of $24 \mu \mathrm{m}$ is soldered into the apex of the base body. The sharp tip apex ensures a small contact radius towards the sample $\left(\mathrm{r}_{\text {tip }}=3 \mu \mathrm{m}\right)$, which promotes a high local resolution. Additionally the comparatively high thermal conductivity of the WC tip $\left(80 \mathrm{Wm}^{-1} \mathrm{~K}^{-1}\right)$, and its close thermocouple attachment yield an effective minimization of the cold finger effect. Herewith connected limiting factors for the spatial resolution and absolute accuracy can usually not be overcome in the same extent within thermopower scanning devices, which use sheath thermocouples or spring loaded tips in direct contact to the sample. Two type T thermocouples (Manufacturer: Labfacility, Model: XF-322-FAR) with a wire diameter of $200 \mu \mathrm{m}$ are used in the setup. While one thermocouple is laser-welded to the body of the scanning tip, the other one is fixed rigidly to the top surface of the ML prior to the measurement using Ag paste. The installation time for the sample sensor takes a couple of minutes for drying of solvent in the Ag paste, whereas scanning of the library is accomplished with a scanning rate of up to $720 \mathrm{~h}^{-1}$ (per measuring point). The scanning tip loads a force of approximately $1.5 \mathrm{~N}$ on the sample surface. The tip contact forms two independent TE measuring circuits, see Fig. 5. The library is mounted with the use of heat conducting paste on a metal block acting as a passive heat sink to ensure isothermal conditions for the entire sample during lift-off periods of the probing tip. The heat sink yields a cold circuit junction at room temperature at the contact point of the sample thermocouple. When the heated probe tip is descended to touch the surface of the ML, a microscopic volume of the thin film is heated near the tip, causing a thermal gradient to spread across the film. The total temperature difference between the sensing points of both thermocouples (see points $\mathbf{M}$ and $\mathbf{C}$ in Fig. 5) amounts typically up to $5 \mathrm{~K}$. The contact radius of the scanning WC 
tip is about $3 \mu \mathrm{m}$, depending on load force and elastic behavior of the sample material. In response to the thermal gradient, a thermovoltage is generated on the length scale of the spread of the thermal field within the film. The thermovoltages $V_{1}$ and $V_{2}$ are measured simultaneously at the $\mathrm{Cu}-\mathrm{Cu}$ and $\mathrm{CuNi}-\mathrm{CuNi}$ wires of the two circuits, respectively, and are evaluated according to the following equations.

$$
\begin{aligned}
& V_{1}=\left(S_{\mathrm{m}}-S_{\mathrm{Cu}}\right)\left(T_{\mathrm{m}}-T_{\mathrm{c}}\right) \\
& V_{2}=\left(S_{\mathrm{m}}-S_{\mathrm{CuNi}}\right)\left(T_{\mathrm{m}}-T_{\mathrm{c}}\right)
\end{aligned}
$$

Here $T_{\mathrm{c}}$ and $T_{\mathrm{m}}$ represent the temperatures of the cold thermocouple junction (sample sensor) and the measured temperature at the probing tip, respectively. $S_{\mathrm{Cu}}$ and $S_{\mathrm{CuNi}}$ are the Seebeck coefficients of $\mathrm{Cu}$ and $\mathrm{CuNi}$, respectively. Combining Eq. (1) and (2), the term $\left(T_{\mathrm{m}}-T_{\mathrm{c}}\right)$ can be eliminated, yielding the Seebeck coefficient of the sample at the contact point

$S_{m}=\frac{V_{1}}{V_{2}-V_{1}}\left(S_{C u}-S_{C u N i}\right)+S_{C u}$

$S_{\mathrm{Cu}}$ and $S_{\mathrm{CuNi}}$ are insensitive to small temperature variations. Due to a small temperature difference of $5 \mathrm{~K}$ during measurement constant values are applied for the wire Seebeck coefficients. Eq. (3) is likewise adopted in simulations and in the PSM measurement to obtain experimental values for $S_{\mathrm{m}}$.

According to the measurement configuration as shown in Fig. 5, the measured thermopower $S_{\mathrm{m}}$ is defined as

$S_{m}=\frac{V_{m}-V_{c}}{T_{m}-T_{c}}$

Here, $V_{\mathrm{m}}$ and $V_{\mathrm{c}}$ represent the electric potential at the points $\mathbf{M}$ and $\mathbf{C}$, respectively. As long as eddy currents in the sample, driven by fluctuations of the thermovoltage, can be neglected, the intrinsic, i.e. physically true, thermopower $S_{\mathrm{i}}$ of the TE film is correlated with the temperatures and potentials at points $\mathbf{S}$ and $\mathbf{C}$ and can be written as

$S_{i}=\frac{V_{s}-V_{c}}{T_{S}-T_{C}}$

Comparing Eqs. (4) and (5), we can see that the discrepancy between $S_{\mathrm{m}}$ and $S_{\mathrm{i}}$ is directly linked to deviations between the measured and the actual temperatures/potentials. This can be quantitatively reproduced by numerical simulation. Here, we define two parameters $r_{\mathrm{t}}$ and $r_{\mathrm{p}}$ as the ratios of the sensed temperature and potential drop, respectively, to the true temperature and potential drop, respectively:

$r_{\mathrm{t}}=\left(T_{\mathrm{m}}-T_{\mathrm{c}}\right) /\left(T_{\mathrm{s}}-T_{\mathrm{c}}\right)$

$r_{\mathrm{p}}=\left(V_{\mathrm{m}}-V_{\mathrm{c}}\right) /\left(V_{\mathrm{s}}-V_{\mathrm{c}}\right)$

Therefore, the relationship between $S_{\mathrm{m}}$ and $S_{\mathrm{i}}$, and the overall relative inaccuracy $r$ can be obtained by 


$$
S_{m}=\frac{r_{p}}{r_{t}} S_{i}
$$

$$
r=\frac{S_{i}-S_{m}}{S_{i}}=1-\frac{r_{p}}{r_{t}}
$$

From Eq. (9) it is clear that the discrepancy between $S_{\mathrm{i}}$ and $S_{\mathrm{m}}$ is influenced by both $r_{\mathrm{t}}$ and $r_{\mathrm{p}}$, which are associated with the heat flow and the temperature and potential distributions in the layered sample structure.

\section{Experimental Results}

Prior to the discussion of the experimental results the difference in spatial resolution of the used characterization techniques is discussed with regard to chemical certainty. The resolution of the Seebeck coefficient measurement is determined primarily by the distribution of thermal gradients within the samples. According to FEM simulations (see Fig. 15) most of the lateral temperature drop is located within $100 \mu \mathrm{m}$ for the experimentally studied substrates. Considering the present average compositional gradients $(0.5$ at. $\% / \mathrm{mm})$ on the ML, the Seebeck coefficient calculated from the thermovoltages of the heated microscopic volume, can be attributed to an area containing a negligible nominal composition spread of approximately 0.125 at.\%o and can thus be considered as taken on a homogeneous sample. As mentioned above, the 4-point probe pins have a distance of 500 $\mu \mathrm{m}$, yielding a nominal composition spread of approximately 0.25 at.\%. The EDX measurement, which is giving the chemical reference for the measured transport properties, offers a relatively high spatial resolution of 1-2 $\mu \mathrm{m}$. However, the average change in composition between equivalent measurement points on the different substrates used is around 2.5 at.\%. Considering an estimated error of $\sim 1$ at. $\%$ the MLs are very similar in composition. According to manufacturer specification the Ambios XP2 profilometer has a vertical resolution between $0.1 \mathrm{~nm}-1 \mathrm{~nm}$, while offering a lateral resolution of $100 \mathrm{~nm}$. Considering the narrow compositional range of the $\mathrm{HH}$-phase and adjacent two phase regions with high thermopower (max. \pm 6 at.\%), the used characterization techniques offer a sufficient resolution to discuss accuracy of thermopower measurement and its interrelation with the distribution of material properties, effects of the substrate material, and the layer thickness.

In order to get a comprehensive overview on the obtained measurement data and to allow correlations between experimental findings and simulation results spatial maps of all experimentally determined data sets are displayed within the supplementary information file. This comprises spatial maps of the element concentration, the Seebeck coefficient, the resistivity, and film thickness. The data sets are shown for each substrate material in direct comparison. The missing data on particular areas of the $\mathrm{Si} / \mathrm{SiO}_{2}$-substrate sample is caused by either delamination or shadowing by the used mask during film deposition. These areas have not been considered for evaluation.

\section{Optical appearance, compositional and thickness distribution of the MLs}

Fig. 1 shows a photograph of each of the fabricated MLs. The optical similarity between the MLs is evident. It is worth noting that the composition spread film deposited on $\mathrm{SiO}_{2} / \mathrm{Si}$ did not adhere as well as on the other substrates in some small areas visible in Fig. 1a. This might be attributed to the higher difference of the thermal expansion coefficient of $\mathrm{SiO}_{2} / \mathrm{Si}$ compared to other substrates in 
relation to the HH-phase. For TiNiSn the averaged linear thermal expansion coefficient is reported between $\alpha_{\mathrm{HH}}=9.6-11.310^{-6} \mathrm{~K}^{-1}[54,55] . \mathrm{SiO}_{2}\left(\alpha_{\mathrm{SiO} 2}=0.3-0.610^{-6} \mathrm{~K}^{-1}[56]\right)$ and $\mathrm{Si}\left(\alpha_{\mathrm{Si}}=2.7-\right.$ $\left.4.210^{-6} \mathrm{~K}^{-1}[57,58]\right)$ have lower values in comparison to $\mathrm{Al}_{2} \mathrm{O}_{3}\left(\alpha_{\mathrm{Al} 2 \mathrm{O} 3}=6.5-8.910^{-6} \mathrm{~K}^{-1}[58,59]\right)$ and $\operatorname{AlN}\left(\alpha_{\text {AlN }}=4.15-5.2710^{-6} \mathrm{~K}^{-1}[58,60]\right)$. The difference of thermal expansion of the substrates might also result in changed thermoelectric properties of the $\mathrm{HH}$ films, as strain can have an effect on the band structure and band gap energy according to reported studies on particular thermoelectric materials [61-63]. A reduction of the thermal conductivity by phonon softening and corresponding reduction of phonon group velocities was likewise demonstrated by application of strain [64-66]. However, it was shown that strain-induced effects show an impact on both, the Seebeck coefficient and the electrical conductivity, yielding a compensating impact on the power factor $\left(P F=S^{2} \sigma\right)$ in many cases [63]. Consequently, and with regard to the present study, similarity of the electrical conductivity measured on different substrates gives sufficient indication for similarity or low level of strain-induced effects on the thermoelectric property distributions on the substrate materials, which allows for a discussion on methodically introduced impacts during thermopower scanning.

Fig. 2 shows the chemical composition distribution of the three MLs in ternary diagrams. Measurement areas that showed spreading under the apertures or peeled off after annealing were excluded. Figure 3 shows the absolute thickness difference of the ML deposited on $\mathrm{SiO}_{2} / \mathrm{Si}$ compared to the libraries deposited on $\mathrm{AlN}$ and $\mathrm{Al}_{2} \mathrm{O}_{3}$. The overall thickness of the ML varies between 250 and $900 \mathrm{~nm}$ with around $500 \mathrm{~nm}$ in the center of the library. The mean thickness difference is $\sim 11 \mathrm{~nm}$ for $\mathrm{SiO}_{2} / \mathrm{Si}$ compared to $\mathrm{AlN}$ and $\sim 23 \mathrm{~nm}$ compared to $\mathrm{Al}_{2} \mathrm{O}_{3}$ as indicated by the red line in the plots of the right side. The thickness difference in both plots show an increase near the center of the library were an increase in the film roughness was observed. The thickness difference can be attributed to the increase surface roughness of the $\mathrm{AlN}$ and $\mathrm{Al}_{2} \mathrm{O}_{3}$ substrates.

\section{Measurements of the electrical resistivity}

The electrical resistivity of the MLs is depicted in a ternary diagram in Fig. 4. The highest values for all MLs lay close to the 1:1:1 compositional region. This is expected since the TiNiSn HH phase is a narrow band-gap semiconductor. A full phase analysis can be found in [15]. The resistivity values range from $0.25 \mu \Omega \mathrm{m}$ to $21 \mu \Omega \mathrm{m}$. The highest resistivity values, between 15 and $20 \mu \Omega \mathrm{m}$, are comparable to the values measured by Jaeger et al., 4-7 $\mu \Omega \mathrm{m}$, for epitaxially deposited TiNiSn [67]. The measurement regions with composition that deviates more than $\sim 5$ at. $\%$ from the 1:1:1 compositional region, generally show a resistivity below $4 \mu \Omega \mathrm{m}$ which indicates metallic behavior. A slight increase in resistivity between $4-6 \mu \Omega \mathrm{m}$ can be identified in all libraries at the very Ni-rich and Sn-deficient part of the ML. Overall the resistivity values are in good agreement with each other.

\section{Results of Seebeck-coefficient measurements}

Thermopower measurements were performed sequentially on the three identical MLs which were deposited on three different substrates. The libraries were measured along parallel lines with a step width of $100 \mu \mathrm{m}$ ( $x$ - and $y$-direction) and under a maximum temperature difference within the sample of $4.5 \mathrm{~K}$. To confirm reproducibility of the measurements each line was scanned two times.

Fig. 6 shows the Seebeck coefficient values of the Ti-Ni-Sn ML deposited on $\mathrm{SiO}_{2} / \mathrm{Si}, \mathrm{AlN}$ and $\mathrm{Al}_{2} \mathrm{O}_{3}$. The stoichiometric TiNiSn phase is the only semiconducting phase in this system. Consequently, the Seebeck coefficient is expected to show a maximum in the central region of the 
ML. All other known phases are expected to be metallic and hence they are expected to show low Seebeck coefficients. The highest Seebeck values were found for compositions of \pm 6 at.\% around the $\mathrm{HH}$ phase, which correspond to a two phase region according to XRD results [15]. A maximum Seebeck coefficient of $-58.6 \mu \mathrm{VK}^{-1}$ was determined for the composition $\mathrm{Ti}_{34} \mathrm{Ni}_{31} \mathrm{Sn}_{35}$ on the $\mathrm{Si}$ substrate, see Fig.6. Compared to stoichiometric TiNiSn, high Seebeck coefficients are measured in the Ni-deficient region on all substrates as well. Around this composition, the Seebeck coefficient monotonically changes to $-15 \mu \mathrm{VK}^{-1}$. The remaining measurement areas show Seebeck values between $-15 \mu \mathrm{VK}^{-1}$ and $5 \mu \mathrm{VK}^{-1}$.

A comparison of the thermopower frequency count spectra of the metallic regions with changing substrate material shows a good accordance between the captured distributions, see Fig. 7. However, a detailed look (see Fig. 10a) reveals positive thermopower values on the Si substrate up to $+5 \mu \mathrm{VK}^{-1}$ compared to small negative values on $\mathrm{Al}_{2} \mathrm{O}_{3}$ and AlN. An opposite sign of the Seebeck values on different substrates could solely be observed on few metallic compositions on the $\mathrm{Si}$ substrate, whereas samples on ceramic substrates $\left(\mathrm{AlN}, \mathrm{Al}_{2} \mathrm{O}_{3}\right)$ showed smooth and consistent courses with equal sign of thermopower. The opposing sing of thermopower on metallic compositions could be possibly attributed to impacts from offset potentials within the measuring circuits. An accordance of the Seebeck coefficient courses on Si and ceramic substrates was confirmed, apart from regions with positive Seebeck coefficient on Si, for every going and returning path of the twofold line-scanned areas. Thus, local variations of the thin film rather than offset potentials could be linked to the observed change of sign. Nevertheless, further studies would be needed to clarify the origin of changed sign of the Seebeck coefficient for these particular sample areas on Si substrate. Furthermore, a clear sequence of absolute thermopower values with respect to the chosen substrate material can be observed on many extended areas of the ML within the thermopower range between $-5 \mu \mathrm{VK}^{-1}$ and $-30 \mu \mathrm{VK}^{-1}$. As shown on an exemplary comparison of line-scans on the right side of Fig. 7, up to $36 \%$ higher absolute thermopower values have been observed for the $\mathrm{Al}_{2} \mathrm{O}_{3}$ substrate compared to the corresponding mean value on the line scans on AlN and Si. The origin of this effect is arising from methodical influences of the PSM probing system related to the thin film measurement, as will be discussed in the simulation and discussion sections in the context of accuracy considerations of thermopower probing.

Another systematic observation for different substrate materials, which is linked to the spatial resolution, relates to the imaging of thermopower transitions at spatial peaks or expanded plateaus and the reproduction of continuous changes of thermopower profiles with multiple reversal points along the scanning direction. Fig. 8 demonstrates that measurements of $\mathrm{HH}$ films on $\mathrm{Al}_{2} \mathrm{O}_{3}$ show a broadening at the base of thermopower peaks, close to thermopower transitions. In comparison to measurements of $\mathrm{HH}$ films on $\mathrm{Si}$ or AlN substrates, this can be observed for pin-like spatial thermopower peaks (see Fig. 8b.) as well as on edges of laterally expanded thermopower plateaus (see Fig. 8a) on $\mathrm{Al}_{2} \mathrm{O}_{3}$. The effect of a reduced spatial thermopower resolution on $\mathrm{Al}_{2} \mathrm{O}_{3}$ becomes likewise visible when multiple changes of thermopower along the scanning direction have to be captured. As shown in Fig. 9, measurements on $\mathrm{Al}_{2} \mathrm{O}_{3}$ reveal much less spatial thermopower features which occasionally can even totally hide narrow local extremal points of the thermopower profiles (see Fig. 10), which could not be observed for measurements of HH films on Si and AlN substrates.

As analytical results on compositions and functional properties have not revealed significant differences for the studied libraries with respect to the chosen substrate, the observed differences in 
measured Seebeck coefficients must be attributed to methodical aspects of the PSM principle. In order to clarify possible sources of uncertainty in accuracy and spatial resolution of thermopower profiles, FEM simulations have been conducted, which will be introduced in the following section.

\section{Simulation results}

\section{Finite element model of the PSM measurement on thin film samples}

To support the interpretation of the obtained PSM results, FEM simulations were carried out using ANSYS ${ }^{\mathrm{TM}}$ based on the schematic shown in Fig. 5. The simulation is based on a three-dimensional (3D) model by setting appropriate symmetry conditions on the center line of the rotationally symmetric probe. The material properties and dimensions that are used in the simulation are given in Table 1. Electrical and thermal properties are assumed to be isotropic for all materials. The surrounding air of the sample is assumed to be adiabatic and no contact resistances are considered. Other parameters, such as the contact radius of the tip $\left(r_{\text {Tip }}=3 \mu \mathrm{m}\right)$ or applied boundary conditions are identical to those in the experimental part. The scanning probe is assumed to be uniformly heated to a constant temperature of $25^{\circ} \mathrm{C}\left(T_{\mathrm{h}}\right)$, while the initial temperature in the whole sample is considered to be constant at $T_{\mathrm{c}} \equiv 20{ }^{\circ} \mathrm{C}$. The potential at the sidewall of the $\mathrm{HH}$ film is set as $V_{\mathrm{c}} \equiv 0 \mathrm{~V}$. In contrast to the experimental part, simulations have been conducted for varying thicknesses of the HH film on each substrate $(0.5 / 1 / 3 / 10 / 25 \mu \mathrm{m})$. The applied node density of the model mesh was set with regard to distances and curvatures of the geometries, and achieved a minimum element length of $100 \mathrm{~nm}$ for the thinnest investigated layer in order to reflect true distributions of temperatures and voltages. $\mathrm{SiO}_{2}$ was studied as an additional possible substrate material since it represents a commonly used wafer material and its thermal conductivity differs significantly from others, which was confirmed to show a strong impact on derived thermopower values. Secondly, simulation of $\mathrm{SiO}_{2}$ as substrate material should give basic access to an assessment of the possible impact of a $\mathrm{SiO}_{2}$ interlayer, which is present on the experimentally studied ML with $\mathrm{Si}$ substrate. An individual case simulation under consideration of a $\mathrm{SiO}_{2}$ interlayer between a $\mathrm{Si}$ substrate and the ML was not conducted within this study. The thermal barrier effect of the interlayer was studied by the simulated case of a complete $\mathrm{SiO}_{2}$ wafer, whereas electrical insulation of the $\mathrm{SiO}_{2}$ was sufficiently reproduced by the low electrical conductivity of $\mathrm{Si}$, which is five orders of magnitude lower than for the HH film.

Initially, simulations have been conducted on homogeneous $\mathrm{HH}$ films in order to capture methodical constrains in determination of correct absolute values for the thermopower and study the effect of the substrate choice and layer thickness of the HH film. Secondly, further simulations were carried out, which took the typical feature of distributed properties on a ML into account. The semiconducting $\mathrm{HH}$ phase was considered as a thin film inclusion of varying width (10 / 25 / 50 / $100 / 300 \mu \mathrm{m}$ ) surrounded by a metallic matrix phase, the physical properties of which correspond to the Ni-deficient, Sn-rich part of the ML (experimental part, see Table 1). Each width of the HH inclusion was simulated for different layer thicknesses and each substrate material, as mentioned before. The Seebeck coefficient of the substrate materials was set to zero, since no impact on the measured thermovoltage is expectable due to the significantly lower electrical conductivity compared to the ML. The measured Seebeck coefficient $S_{\mathrm{m}}$ was derived in analogy to the real experiment and in accordance to Eq. (3), by evaluation of the temperature and voltage readings at the place of real signal tapping close to the apex of the scanning tip.

Table 1. Material properties and geometrical dimensions of the FE model for PSM 
measurement simulations. $d$ : film thickness/height, $w$ : width of the $\mathrm{HH}$ inclusion, $\sigma$. electrical conductivity, $\kappa$ : thermal conductivity, $S_{\mathrm{i}}$ : assigned Seebeck coefficient.

\begin{tabular}{|c|c|c|c|c|c|}
\hline Material & $d[\mu \mathrm{m}]$ & $w[\mu \mathrm{m}]$ & $\sigma[\mathrm{S} / \mathrm{cm}]$ & $\kappa[\mathrm{W} / \mathrm{K} \cdot \mathrm{m}]$ & $S_{\mathrm{i}}[\mu \mathrm{V} / \mathrm{K}]$ \\
\hline $\mathrm{Si}$ & 400 & 400 & $1.56 \times 10^{-5}$ & $150[68]$ & 0 \\
\hline $\mathrm{Al}_{2} \mathrm{O}_{3}$ & 400 & 400 & $1 \times 10^{-16}$ & 36.8 [69] & 0 \\
\hline AlN & 400 & 400 & $1 \times 10^{-16}$ & 180 [70] & 0 \\
\hline $\mathrm{SiO}_{2}$ & 400 & 400 & $1 \times 10^{-16}$ & $6.5[71]$ & 0 \\
\hline HH phase & $0.5-25$ & 400 & 400 & 10 & -50 \\
\hline HH inclusion & $0.5-25$ & $10-300\left(w_{\text {inc }}\right)$ & 400 & 10 & -50 \\
\hline Matrix & - & $100-390$ & $2 \times 10^{3}$ & 20 & 4 \\
\hline $\mathrm{TC}$ & 20 & $3\left(r_{\text {Tip }}\right)$ & $50 \times 10^{3}$ & 80 & 6 \\
\hline $\mathrm{Cu}$ & - & 40 & $625 \times 10^{3}$ & 400 & 2 \\
\hline
\end{tabular}

Absolute accuracy of PSM thermopower measurements on homogeneous half-Heusler thin films

As heat passes through the probe to the sample, a temperature difference $\Delta T_{1}$ is inherently generated in the tip, which equals the temperature deviation between the measured (point $\mathbf{M}$ ) and the real (point $\mathbf{S}$ ) hot-side temperature of the HH film $\left(\Delta T_{1}=T_{\mathrm{m}}-T_{\mathrm{s}}\right)$. The deviation is determined by the fraction of the thermal resistance located between these points, in relation to the overall thermal resistance of the configuration, and yields a principal underestimation of the measured thermopower. The heat flow scales inversely with the total thermal resistance and is consequently sensitive to the choice of the substrate material and layer thickness of the HH film. For a low layer thickness the total heat flow amounts up to $8.12 \mathrm{~mW}$ for the highest conductive substrate (AIN) and decreases to approximately $1 \mathrm{~mW}$ for the lowest conductive substrate material under test $\left(\mathrm{SiO}_{2}\right)$, see Fig. 11 . Different thermal properties of the ML and substrate yield the heat flow to be not uniformly distributed between layers and consequently it is reasonable to distinguish between cross- and in-plane heat conduction for different sample configurations, in order to assess individual signal contributions and particular error sources.

For substrates with higher thermal conductivity the total heat flow decreases with increasing film thickness of the ML (see $\mathrm{Si}, \mathrm{AlN}$, and $\mathrm{Al}_{2} \mathrm{O}_{3}$ in Fig. 11) as the sample heat transport is carried to a large part by cross- and in-plane conduction within the highly conductive substrates. The modulation of the heat flow by different layer thicknesses of the HH films is consequently strongest for highly conductive substrates. Since the cross-plane temperature drop over the HH film increases with increasing thickness (see Fig. 12), a reduction of the heat flow within the substrate is observed. Due to the lower thermal conductivity of the $\mathrm{HH}$ film, the in-plane heat flow within the film increases to a smaller extent for higher thickness. This finally yields an asymptotical approximation of the total heat flow with a final value converging to $2 \mathrm{~mW}$ for high film thickness and substrates with higher thermal conductivity compared to the $\mathrm{HH}$ film. In case of a lower thermal conductivity 
of the substrate (see $\mathrm{SiO}_{2}$ in Fig. 11), in-plane conduction within the $\mathrm{HH}$ film represents the major heat transport within the sample, as the total heat flow slightly increases with higher film thicknesses, which is contrary to all other studied substrates. This is confirmed likewise by the significantly lower cross-plane temperature difference for the $\mathrm{HH}$ film on $\mathrm{SiO}_{2}$, which is five- or tenfold smaller than on $\mathrm{Al}_{2} \mathrm{O}_{3}$ or Si/AlN for thinner layers, respectively (see Fig. 12).

The varying incident heat flow yields different temperature deviations $\Delta T$ and consequently the absolute value of the measured thermopower is altered differently for the studied sample configurations, as can be seen from Fig. 13. According to obtained simulation results the reduction of the measured Seebeck coefficient below the true value is between $5 \%$ and $50 \%$, depending on the thickness and substrate choice. In order to understand the origin of the discrepancy, the temperature and potential deviation ratios $\left(r_{\mathrm{t}}\right.$ and $\left.r_{\mathrm{p}}\right)$ for all samples are plotted in Fig. 14. As can be seen for the highest conductive substrates (AIN, $\mathrm{Si}$ ) $r_{\mathrm{t}}$ is observed to dominate the discrepancy of $S_{\mathrm{m}}$, which is caused by the considerably higher heat flow and corresponding temperature drop along the tip apex. Due to increased heat flow a still remarkable potential drop results from the temperature drop at the tip apex related to the assumed non-negligible thermopower value of the tungsten carbide tip. Overall, one must expect large discrepancies of $S_{\mathrm{m}}$ between $15 \%$ and $50 \%$ if $\mathrm{AlN}$ or $\mathrm{Si}$ is used as the substrate. A similar but attenuated situation in terms of uncertainty occurs for $\mathrm{Al}_{2} \mathrm{O}_{3}$. A deviation for $S_{\mathrm{m}}$ between $13 \%$ and $26 \%$ is calculated here, likewise being dominated rather by the temperature deviation $r_{\mathrm{t}}$ than by $r_{\mathrm{p}}$. The best reproduction of the intrinsic thermopower of the $\mathrm{HH}$ film is observed for $\mathrm{SiO}_{2}$ as a substrate material. Due to the low heat flow both, $r_{\mathrm{t}}$ and $r_{\mathrm{p}}$ are close to unity yielding an overall deviation for $S_{\mathrm{m}}$ between $5 \%$ and $8 \%$. This analysis clearly explains why the measurements on higher conductive substrates yield larger discrepancies of $S_{\mathrm{m}}$ on all film thicknesses. Moreover, the simulation shows that the measurement inaccuracy of the PSM is quite large when the film thickness is very small. It could be reduced to $\leq 15 \%$ by increasing the film thickness above $25 \mu \mathrm{m}$ on higher conductive substrates, which is not feasible in thin film combinatorial materials science. Therefore, the use of thermally lower conductive $\mathrm{SiO}_{2}$ as a substrate material is recommended, where the inaccuracy could be reduced to $<8 \%$, also for thin films.

Spatial resolution and accuracy of the PSM applied on inhomogeneous TFCS with laterally distributed properties

The simulation results shown above address the expectable accuracy of the Seebeck coefficient on a spatially extended and homogeneous $\mathrm{HH}$ thin film for different thickness and substrate materials. However, a statement on the absolute accuracy and the ability to hide a potentially interesting semiconducting phase on a ML requires the consideration of adjacent sample regions with possibly differing TE properties. It is assumed, that the worst case for proper indication of correct thermopower values is given by a spatial peak function of a spot-shaped semiconducting phase surrounded by metallic material, as this configuration is expected to distort temperature and potential distributions significantly. In this context, rather the effective in-plane temperature distribution dominates the resulting deviation of thermopower since cross-plane temperature differences turned out to be smaller. Secondly, no impact on the electrical field can be expected from the substrates, as they have very low electrical conductivity and zero thermopower was assigned to them. In order to understand the intrinsic spatial resolution for the studied cases, further evaluations of the in-plane temperature profiles within the layered sample structure were carried out for the homogeneous $\mathrm{HH}$ phase initially. 
Fig. 15 shows that in-plane temperature profiles within the $\mathrm{HH}$ film differ significantly for different substrates and layer thicknesses. Generally, the lower the thermal conductivity of the substrate the farther becomes the radius of the in-plane temperature field in the $\mathrm{HH}$ film. However, results for substrates with higher thermal conductivity than the $\mathrm{HH}$ film $\left(\mathrm{Al}_{2} \mathrm{O}_{3}, \mathrm{Si}\right.$, AlN) exhibit in the far field a remarkable sequence of absolute in-plane temperatures with respect to the film thickness, which is contrary to substrates with a lower thermal conductivity than the HH film. Although the in-plane thermal resistance of the $\mathrm{HH}$ film increases with decreasing layer thickness, the highest in-plane temperatures and spread of the temperature field can be observed in the far field for the thinnest (i.e. $0.5 \mu \mathrm{m}) \mathrm{HH}$ films on thermally highly conductive substrates. This points out to a dominance of the substrate heat flow over the in-plane temperature profile within the $\mathrm{HH}$ film, particularly for thin films. In this case the lateral temperature drop within the substrate is projected onto the in-plane thermal field of the $\mathrm{HH}$ film. The in-plane temperature profile changes with respect to film thickness for low conductive substrates $\left(\mathrm{SiO}_{2}\right)$, Fig. 15. Here, almost no heat flow passes the substrate and consequently the thermal spread and absolute temperatures of the in-plane field increase for higher film thickness.

Highest temperature gradients within the $\mathrm{HH}$ film are principally located near the tip, and consequently the near field regions contribute most to the thermopower signal. Generally, higher conductive substrates yield the spatially narrowest gradient zone, as the heat is dissipated more effectively into the substrate. However, the configuration faces a competing situation between the in-plane conduction within the $\mathrm{HH}$ film and the cross-plane heat dissipation through the $\mathrm{HH}$ film into the substrate. Both paths of heat flow are affected by the HH film thickness as well as by the thermal conductivity of the substrate and determine the shape of the temperature gradient in the near field, which predominantly governs the spatial resolution of a thermopower measurement on a ML.

For higher conductive substrates, a strongly focused temperature gradient with relatively broad but shallow foothills is observed in-plane. Lower in-plane gradients are stimulated by the heat sink action of the substrate whereas the foothills scale inversely proportional to the thermal conductivity of the substrate, as its lateral temperature drop is projected onto the upper $\mathrm{HH}$ film. The lower the thermal conductivity of the substrate, the steeper and wider these foothills will get (compare results for $\mathrm{Al}_{2} \mathrm{O}_{3}$ on the one hand and $\mathrm{AlN}$ and $\mathrm{Si}$ on the other, in Fig. 15). As the film thickness increases from $0.5 \mu \mathrm{m}$, the imprint of the lateral substrate temperature profile onto the top film is washed out step by step. This, in effect, will let thermal gradients increase initially in the near field and causes a narrowing of shallow thermal foothills next to this region. The situation changes with continuous increase of film thickness. Then, the thermal near field is determined by the direct in-plane heat flow within the top film due to decreasing heat dissipation into the substrate and weakened imprint of the substrate temperature profile onto the film. This results in a reversal point of the evolution of temperature gradients and width of the adjacent foothills. The thermal gradient starts to decrease again on $\mathrm{Al}_{2} \mathrm{O}_{3}$ above $10 \mu \mathrm{m}$ top layer thickness whereas, due to higher thermal conductivity, the point of return is already reached between $3 \mu \mathrm{m}$ and $10 \mu \mathrm{m}$ on Si and AlN, see Fig. 15.

In order to develop an assessment of the spatial resolution of thermopower mapping, the effective width of in-plane temperature differences was evaluated from simulation results. The evaluation considers a radius that covers $70 \%$ and $90 \%$, respectively, of the total in-plane temperature difference within the $\mathrm{HH}$ films for each sample configuration (see Fig. 16). For a low thermally conducting substrate $\left(\mathrm{SiO}_{2}\right)$, results show a strictly monotonic increase of the radius with increasing 
film thickness. As almost no heat is conducted by the substrate in this case, an increase of layer thickness yields a monotonic decrease of thermal resistance which leads to a broadening of the lateral thermal field from approximately $80 \mu \mathrm{m}$ to $110 \mu \mathrm{m}$ on $\mathrm{SiO}_{2}(70 \%$ radius). The reversal point for the evolution of gradients with respect to layer thickness shows a non-monotonic dependence on higher conducting substrates and consequently best lateral resolution for a particular layer thickness. According to the modeling results, an optimal resolution is expected for a film thickness around $3 \mu \mathrm{m}$ on $\mathrm{Si}$ or $\mathrm{AlN}$ and for $10 \mu \mathrm{m}$ film thickness on $\mathrm{Al}_{2} \mathrm{O}_{3}$. It is found that a considerably wide film region contributes potentially to the thermovoltage signal even on higher conductive substrates. While $70 \%$ of the in-plane temperature difference within the top layer drops over a radius between $20 \mu \mathrm{m}$ and $60 \mu \mathrm{m}$ for $\mathrm{Al}_{2} \mathrm{O}_{3}$, this range can be even up to $30 \mu \mathrm{m}$ on $\mathrm{Si}$ and $\mathrm{AlN}$, despite the fivefold higher thermal conductivity. The $90 \%$ radius reaches values significantly above $200 \mu \mathrm{m}\left(\mathrm{Al}_{2} \mathrm{O}_{3}\right.$, $\mathrm{SiO}_{2}$ ) and still between $25 \mu \mathrm{m}$ and $175 \mu \mathrm{m}$ for highest conductive substrates under test ( $\left.\mathrm{Si}, \mathrm{AlN}\right)$.

These values give a measure of the sensing radius of the Seebeck probing. Sample regions of different material properties in the vicinity of the contact point start to contribute to the signal when they are within the radius of the gradient zone. In contrast to the previously discussed case of a homogeneous $\mathrm{HH}$ top layer, the assumption of a surrounding metallic matrix will lead to a reduction of the effective in-plane temperature difference across the semiconducting inclusion. This reduction will not be detected by the temperature sensor at the scanning tip, and consequently comparably lower thermovoltages will be divided by an overestimated temperature difference, resulting in a further underestimation of thermopower additionally to the cold finger effect on a homogeneous film. The decline of the effective in-plane temperature difference over the semiconducting phase propagates further with decreasing width of the inclusion. A second effect on the measured thermopower is observed for small inclusions where cross-plane temperature differences cover also the surrounding metal phase. Electrically, this can be considered as a closed circuit of two voltage sources, according to the different e.m.f. generated by the semiconductor and the metal, respectively. The parallel arrangement in the temperature gradient results in a net thermovoltage driving eddy currents which are balanced by the Ohmic voltage drop along closed current loops crossing the boundary between the adjacent different sample regions twice. Thus, the terminal voltage, which is used to calculate the measured thermopower, is reduced since the Ohmic contribution is counteracting the thermo-emf of the semiconductor, which is observable similarly for measurements on inhomogeneous bulk samples [46].

Compared to the homogeneous sample this obviously leads to an additional falsification of thermopower assigned to a particular scanning point on the semiconducting phase. The width of the $\mathrm{HH}$ inclusion (see Fig. 5) plays an important role for the achievable measurement accuracy and ultimately the capability for the recognition of spatially narrow thermopower peaks. The resulting impact on the deducible absolute thermopower value was determined in accordance to the model description within the simulation section. The simulation reflects a thermopower measurement in the middle of a particular inclusion and sets the measured thermopower into relation to the corresponding measurement (same substrate and film thickness) on the homogeneous sample. Fig. 17 illustrates the outcome of the FEM simulation for all sample configurations. As an inclusion width of $300 \mu \mathrm{m}$ exceeds the maximal in-plane sensing radius, the thermopower will equalize the homogeneous case, independently of the substrate material being used. However, reduction of width will rise essential differences in thermopower accuracy with respect to the chosen substrate material and film thickness of the ML. The effective heat dissipation of high conductive substrates (Si or 
AlN) keeps the additional thermopower error $<10 \%$ for inclusion widths down to $100 \mu \mathrm{m}$ and film thicknesses $>1 \mu \mathrm{m}$. Due to expanded in-plane temperature fields, lower conductive substrates $\left(\mathrm{Al}_{2} \mathrm{O}_{3}, \mathrm{SiO}_{2}\right)$ double the expectable error in the same sample configuration. With inhomogeneity widths between $100 \mu \mathrm{m}$ and $300 \mu \mathrm{m}$ the additional error is attributed dominantly to the decrease of in-plane temperature difference over the inclusion. Further reduction of the inclusion width yields an increase of additional thermopower errors on all substrates. However, for lower film thicknesses, $\mathrm{Si}$ and AlN reveal a maximal error increase of approximately $40 \%$ to $45 \%$, whereas $\mathrm{Al}_{2} \mathrm{O}_{3}$ and $\mathrm{SiO}_{2}$ exhibit additional errors above $60 \%$ and $70 \%$, respectively.

Again, the higher the thermal conductivity of a substrate, the higher will be the modulation of the error level with respect to film thickness. The low heat dissipation into the $\mathrm{SiO}_{2}$ substrate keeps the error increase small for any width of inclusion. In the light of low cross-plane temperature difference on this substrate, the dominant origin of additional error by the inhomogeneity remains to be related to the decrease of effective in-plane thermal gradients over the semiconducting phase. Higher conductive substrates show significantly higher sensitivity to changes of the film thickness. In accordance to these characteristics the error increase for an inhomogeneous sample behaves non-monotonic for inclusions widths $<50 \mu \mathrm{m}$ resembling the characteristics of the $70 \%$ radius on highly conductive substrates. For smallest inclusions of $10 \mu \mathrm{m}$ this yields for $\mathrm{Si}$ and AlN a minimum additional error close to $3 \mu \mathrm{m}$ film thickness, which amounts up to $18 \%$ and $16 \%$, respectively. Due to broadening of the thermal field the corresponding error increase for lower conductive substrates reveals to be by far higher and amounts to $44 \%\left(\mathrm{Al}_{2} \mathrm{O}_{3}\right)$ and above $70 \%$ $\left(\mathrm{SiO}_{2}\right)$. Finally, a remarkable increase of thermopower uncertainty is observable on Si and AlN for the smallest studied inclusions and at higher film thickness $(>5 \mu \mathrm{m})$. Since the impact of the substrate material on the thermal field distribution is decreasing successively with increasing film thickness, this yields an increase of the cross-plane temperature difference over the film. The reason for increased error on small inclusions and for higher film thickness is given by the occurrence of eddy currents between the dissimilar phases in the film that are thermally aligned in parallel. This leads to significantly lowered thermovoltages and an increased underestimation of corresponding thermopower.

\section{Discussion}

Both FEM simulations and PSM experiments reveal systematic differences in the measured thermopower on $\mathrm{HH}$ thin film libraries with respect to the chosen substrate material. Experiments indicate improved conditions for the detection of narrow pin-like thermopower peaks on better conducting substrates $\left(\mathrm{Si}, \mathrm{AlN}\right.$ ) compared to $\mathrm{Al}_{2} \mathrm{O}_{3}$ (see Fig. 10) as it was predicted by FEM simulations. Compared to $\mathrm{Al}_{2} \mathrm{O}_{3}$ a better imaging of transitions can be achieved likewise on spatially expanded plateaus if Si or AlN are chosen as substrates since base levels of spatial Seebeck pulses are blurred to a lower extent than on $\mathrm{Al}_{2} \mathrm{O}_{3}$ (see Fig. 8b). The weaker cold finger effect on $\mathrm{Al}_{2} \mathrm{O}_{3}$ yields a better absolute accuracy of thermopower in homogeneous regions of a ML, which was qualitatively confirmed likewise by experiments and simulations (see Figs. 7,8,9). According to simulation, PSM measurements on $\mathrm{Si}$ and $\mathrm{AlN}$ should show a thermopower of approximately $70 \%$ of that on $\mathrm{Al}_{2} \mathrm{O}_{3}$ for a homogeneous film with a thickness of $0.5 \mu \mathrm{m}$. In fact, many areas of the measured ML reveal similar thermopower differences in comparison of these substrates, as is shown for instance in Fig. 9a. For Si and AlN, the peak value of thermopower at the plateau amounts to approximately $64 \%$ of the corresponding value on $\mathrm{Al}_{2} \mathrm{O}_{3}$, in good agreement to simulation. 
However, the degree of accordance between simulation and experiment varies over the studied libraries. As can be seen from Figs. 7-9 the order of thermopower values with respect to the substrate is repeating on many areas of the ML, but does not coincide quantitatively to the prediction by simulation. The discrepancy between simulated and measured thermopower ratios with respect to the substrate material is likewise observed for higher conductive substrates. According to simulation, measurements of thermopower on Si and AlN should show only minor differences among each other. The thermally low conducting $1.5 \mu \mathrm{m}$ thick $\mathrm{SiO}_{2}$ layer on top of the Si substrate, which was not considered for FEM simulation, could alter the temperature distribution (in- and cross-plane) within the sample and lower the incident heat flow from the scanning tip, which effectively would yield a higher similarity of measurement results on $\mathrm{Si}$ and $\mathrm{Al}_{2} \mathrm{O}_{3}$ substrates. Such accordance was observed solely on few sample areas of the MLs (Fig. 7b). In contrast, the small base width of pin-like thermopower peaks measured on Si substrate indicates a contrary picture, which is more in accordance with the interpretation from FEM simulations. In fact, experimental findings exhibit deviations from simulation results on multiple areas of the libraries but with no systematic correlation and without support of findings from simulation results.

Such differences can arise from variations of the tip contact area, film thickness, and thermal resistance at the contact spot as well as from locally varying thermal interface resistance between a substrate and the film. Since multiple repeated measurements on same areas of the ML have been performed without noticeable shifts of thermopower, one can assume $\mathrm{HH}$ thin film samples to be insensitive to plastic deformation, as an effect of high material hardness. Consequently, the thermal coupling between the scanning tip and the sample surface in terms of the effective contact area is considered as constant among the measurements on different sample configurations since no traces of plastic deformation have been observed on the surface of the $\mathrm{HH}$ thin films. According to measurements on the $\mathrm{HH}$ ML, the average thickness was determined to $575 \mathrm{~nm}$ with a standard deviation of $164 \mathrm{~nm}$. In relation to the sensitivity of thermopower measurements upon the film thickness, a deviation of less than 5\%pts is expected within a thickness range from $0.5 \mu \mathrm{m}$ to $1 \mu \mathrm{m}$ (see Fig. 13b.). Obviously, this relation does not hold for the observed differences between thermopower results on $\mathrm{Si}$ and $\mathrm{AlN}$, as they often show much higher differences and occasionally even different sign of thermopower (only observed on metallic phases of the ML - see Fig. 10a.), which cannot be due to layer thickness, but either to an elusive impact of electrical offset potentials within the measurement circuits. Furthermore, variations of the film thickness revealed a good accordance between individual depositions on different wafers, which yields a good accordance of the electrical conductivity data. The effect of a non-ideal electrical insulation between a semiconducting layer and an electrically conductive substrate (here: $\mathrm{Si}$ ) could principally hold for a significant impact on the measured thermopower as could be shown in a previous work on electrodeposited $\mathrm{Bi}_{2} \mathrm{Te}_{3}$ films on $\mathrm{Au}$ substrates [72]. Electrical crosstalk to the substrate by imperfections of the overlying $\mathrm{SiO}_{2}$ layer must have led to corresponding deviations of the electrical measurements, which could not be observed. Thus, electrical substrate impact can be likewise excluded as a possible cause of observed thermopower differences on Si and AlN substrates. Subjected to the requirement of solely dissimilar substrate materials a weak similarity of the thermal coupling between HH-films and studied substrate materials can be given accordingly as a reason for the observed discrepancy between simulations and measurements on the ML. While simulations assumed a perfect thermal contact between the top layer and the substrate, the introduction of a thermal contact resistance would alter the distributions of temperature gradients, effectively lowering the impact of a particular substrate material on the thermopower measurement. First signs 
of possible differences in thermal coupling between substrate and film could be observed by partial delamination on metallic phases outside in the central region of the wafer. Since properties of the thermal coupling within the samples could not be accessed within this study, further investigation on the similarity of thermal contact resistances within the samples should be undertaken. Although the direct determination of thermal contact properties is difficult, an indirect access could be by adhesion tests and, assuming similar material compositions, by preparation of several libraries on different substrates, varying the layer thickness of the deposited $\mathrm{HH}$ libraries as it was studied by simulations in this work.

Finally, a possible influence of different microstructures on different substrates on the measured data should be considered. Microstructure has indeed an impact on the thermoelectric properties of the TiNiSn-System. Four ternary phases have been verified on the deposited ML in a previous work [15]. Beside the cubic half-Heusler phase TiNiSn, the full-Heusler phase $\mathrm{TiNi}_{2} \mathrm{Sn}$, the hexagonal phase $\mathrm{Ti}_{5} \mathrm{NiSn}_{3}$ and the tetragonal phase $\mathrm{Ti}_{2} \mathrm{Ni}_{2} \mathrm{Sn}$ were found. The TiNiSn-phase has a small compositional area in which it is stable as a single phase. Measurement areas on the ML with an off-stoichiometry of 1-3 at\% were found to be already two phase regions. However, with regard to the discussion of impacting factors on the determination of local thermopower similarity of the deposited $\mathrm{HH}$ films comes to the fore, which is ensured in this work experimentally by the simultaneous processing of MLs under inert conditions (deposition and annealing) and proven by the good agreement of the electrical conductivity data obtained on all studied samples. The accordance of the electrical conductivity minimizes a possible impact of differences in grain size, orientation, density, oxygen contamination and film stress among the studied MLs. Since local distributions and variations of these parameters, or generally microstructure features have not been considered with their corresponding impact on the thermopower within simulations, measurement results could be possibly not fully reproduced quantitatively. Only few experimental data are available on thin film TiNiSn in literature to date. First thin film preparation and corresponding investigations on HH-TiNiSn are dated from 2011 [73,74]. Consequently, further experiments and measurements have to be accomplished in order to clarify additional influencing parameters on the thermopower of TiNiSn thin films, especially with regard to the discussion on employable characterization techniques and their accuracy.

\section{Conclusions}

Combinatorial thin film ML of the Ti-Ni-Sn system were fabricated on different substrates by magnetron sputtering. Spatially resolved measurements of the Seebeck coefficient have been performed on these libraries by the Potential \& Seebeck Microprobe, revealing significant differences in absolute values of thermopower with respect to the chosen substrate material. Corresponding FEM simulations confirmed the substrate impact on temperature and potential fields and hence, due to a spurious temperature difference within the sensing probe and TE eddy currents in inhomogeneous films, on the measured Seebeck coefficient. Simulation reveals that thermally lower conducting substrates yield a better absolute accuracy of thermopower on homogeneous films. In a simple film-on-substrate configuration, simulations show a quite large inaccuracy of the PSM measurements for low film thickness $(\mu \mathrm{m})$ that can be significantly reduced to $\leq 15 \%$ for film thickness $>25 \mu \mathrm{m}$ on higher conductive substrates, and further reduced to $<8 \%$ by using thermally low conductive $\mathrm{SiO}_{2}$ as substrate. Furthermore, lowly conductive substrates yield a weaker modulation of the measured thermopower with respect to layer thickness, i.e. measurements on such libraries are less sensitive to uncertainty or variation of the film thickness. The choice of a particular 
substrate material alters the dimension of temperature fields within the sample and determines consequently the accuracy of measured thermopower. Since the compositional gradient on a ML is linked to diffusion processes and the inherent constitutional phase width within a phase diagram, the ability to recognize a particular composition in terms of TE performance becomes a matter of the used substrate, too. Imaging of thermopower transitions like in the case of line phases is facilitated by thermally highly conductive substrates, since better heat dissipation yields a narrowing of lateral temperature gradients, effectively lowering the impact of inhomogeneous thermopower distributions within the sample on the scan profile shape. Simulation results of this work can only provide estimates on accuracy for particular measurement conditions that are determined methodically by the tip contact area, employed materials and assumed geometries of the studied configurations, and which were set with best possible match to true measurement conditions. Quantitative conclusions on the accuracy are limited to the validity of further measurement conditions and simulation assumptions, which must be seen under the light of unconsidered and potentially varying thermal contact properties (tip-film / film-substrate), which have a distinct effect on the measured thermopower and may explain the observed occasional inconsistencies between simulation and experiment. Nevertheless, qualitative results of this study point to a specific maximum lateral resolution for local thermopower mapping on higher conductive substrates with respect to the applied film thickness of the deposited libraries. Maximum lateral resolution can be achieved on $\mathrm{Si}$ and $\mathrm{AlN}$ substrates at a film thickness around $3 \mu \mathrm{m}$, while $\mathrm{Al}_{2} \mathrm{O}_{3}$ shows its maximum for about $10 \mu \mathrm{m}$ thick films. For thermopower probing of combinatorial thin film ML, it can be concluded in summary, that unknown material systems which eventually contain semiconducting line phases along with adjacent metallic phases should be characterised first on thermally well-conducting substrates with adapted layer thickness for recognition of high performance phases on a wide compositional scale. In a second step, narrow constitutional intervals containing interesting compositions should be deposited on thermally less conductive substrates in order to verify the observed thermopower correlation to the materials structure and to achieve best accuracy of the Seebeck coefficient for candidate high performance phases.

\section{Acknowledgments}

The authors gratefully acknowledge the financial support from the German Research Foundation (DFG) for funding of the project "Knowledge-based design and screening of thermoelectric half-Heusler alloys using high-throughput computations and experiments" under the DFG grant numbers MU1651/5-1 and LU1175/9-1.

\section{References}

[1] Sootsman, J.R.; Chung, D.Y.; Kanatzidis, M.G. New and old concepts in thermoelectric materials. Angew. Chem. Int. Edit. 2009, 48, 8616-8639.

[2] NASA, Project description: "Advanced Low Temperature Thermoelectric Materials for Cryogenic Power Generation Project”, http://techport.nasa.gov/view/16820, Retrieved 2016-05-15.

[3] Lal, S.; Loo, S.; Chung, D.-Y.; Kyratsi, T.; Kanatzidis, M. G.; Cauchy, C.; Hogan, T.P. Thermoelectric module for low temperature applications. Mat. Res. Soc. Symp. Proc. 2002, 691, G6.2.1 - G6.2.9.

[4] Rowe, D. M. General Principles and Basic Considerations. In Thermoelectric Handbook Macro to Nano, Rowe, D. M., Eds.; Boca Raton, USA: Taylor \& Francis Group, 2006, pp. 1.1 - 


\subsection{5, DOI: 10.1201/9781420038903.sec1.}

[5] Zhao, Y.; Dyck, J.S.; Hernandez, B.M.; Burda, C. Enhancing Thermoelectric Performance of Ternary Nanocrystals through Adjusting Carrier Concentration. Journal of the American Chemical Society 2010, 132, 4982-4983.

[6] Hsu, C.; Liu, Y.; Ma, H. Effects of the $\mathrm{Zr}_{0.5} \mathrm{Hf}_{0.5} \mathrm{Fe}_{\mathrm{x}} \mathrm{Co}_{1-\mathrm{x}} \mathrm{Sb}_{0.8} \mathrm{Sn}_{0.2} / \mathrm{Fe}_{3} \mathrm{Sn} 2$ half-Heusler composites on the ZT value. Journal of Alloys and Compounds 2015, 621, 324-330.

[7] Dresselhaus, M.S.; Chen, G.; Tang, M.Y.; Yang, R.G.; Lee, H.; Wang, D.Z.; Ren, Z. F.; Fleurial, J. P.; Gogna, P. New Directions for Low-Dimensional Thermoelectric Materials. Advanced Materials 2007, 19, 1043-1053.

[8] Nolas, G. S.; Morelli, D. T.; Tritt, T. M. Skutterudites: A Phonon-Glass-Electron Crystal Approach to Advanced Thermoelectric Energy Conversion Applications. Annu. Rev. Mater. Sci. 1999, 29, 89-116.

[9] Dadda, J.; Müller, E.; Perlt, S.; Höche, T.; Bauer Pereira, P.; Hermann, R. P. Microstructures and nanostructures in long-term annealed $\mathrm{AgPb}_{18} \mathrm{SbTe}^{20}$ (LAST-18) compounds and their influence on the thermoelectric properties. J. Mater. Res. 2011, 26, 1800-1812.

[10]Venkatasubramanian, R.; Siivola, E.; Colpitts, T.; O`Quinn, B. Thin-film thermoelectric devices with high room-temperature figures of merit. Nature 2001, 413, 597-602.

[11]Li, J.F.; Liu, W.S.; Zhao, L.D.; Zhou, M. High-performance nanostructured thermoelectric materials. NPG Asia Materials 2010, 2, 152-158.

[12]Hsu, K.F., Loo, S., Guo, F., Chen, W., Dyck, J.S., Uher, C., Hogan, T., Polychroniadis, E.K., Kanatzidis, M.G. Cubic $\mathrm{AgPb}(\mathrm{m}) \mathrm{SbTe}(2+\mathrm{m})$ : bulk thermoelectric materials with high figure of merit. Science 2004, 303, 818-821.

[13]Chen, Z.G.; Han, G.; Yang, L.; Cheng, L.; Zou, J. Nanostructured thermoelectric materials: Current research and future challenge. Progress in Natural Science: Materials International 2012, 22, 535-549.

[14]Nolas, G.S.; Sharp, J.; Goldsmid, J., Thermoelectrics - Basic Principles and New Materials Developments; Springer: Berlin-Heidelberg, 2001; pp 177-207.

[15]Wambach, M.; Stern, R.; Bhattacharya, S.; Ziolkowski, P.; Müller, E.; Madsen, G.K.H.; Ludwig, A. Unraveling Self-Doping Effects in Thermoelectric TiNiSn Half-Heusler Compounds by Combined Theory and High-Throughput Experiments. Advanced Electronic Materials 2015, 2, 1500208.

[16]Uher, C.; Yang, J.; Hu, S.; Morelli, D. T.; Meisner, G. P. Transport properties of pure and doped MNiSn (M=Zr, Hf). Phys. Rev B 1999, 59, 8615.

[17]Hohl, H.; Ramirez, A. P.; Goldmann, C.; Ernst, G.; Wölfing, B.; Bucher, E. Efficient dopants for ZrNiSn-based thermoelectric materials. J. Phys.: Condens. Matter 1999, 11, 1697-1709.

[18] Sakurada, S.; Shutoh, N. Effect of Ti substitution on the thermoelectric properties of (Zr,Hf)NiSn half-Heusler compounds. Appl. Phys. Lett. 2005, 86, 082105.

[19]Chen, S.; Lukas, K. C.; Liu, W.; Opeil, C. P.; Chen, G.; Ren, Z.F. Effect of Hf Concentration on Thermoelectric Properties of Nanostructured N-Type Half-Heusler Materials $\mathrm{Hf}_{\mathrm{x}} \mathrm{Zr}_{1-}$ ${ }_{x} \mathrm{NiSn}_{0.99} \mathrm{Sb}_{0.01}$. Adv. Ener. Mat. 2013, 3, 1210.

[20] Schwall, M.; Balke, B. Phase separation as a key to a thermoelectric high efficiency. Phys. Chem. Chem. Phys. 2013, 15, 1868-1872.

[21]Yu, C.; Zhu, T. J.; Shi, R. Z.; Zhang, Y.; Zhao, X. B.; He, High-performance half-Heusler thermoelectric materials $\mathrm{Hf}_{1-x} \mathrm{Zr}_{x} \mathrm{NiSn}_{1-y} \mathrm{Sb}_{y}$ prepared by levitation melting and spark plasma sintering. J. Acta Mater 2009, 57, 2757-2764. 
[22] Green, M. L.; Takeuchi, I.; Hattrick-Simpers, J. R. Applications of high throughput (combinatorial) methodologies to electronic, magnetic, optical, and energy-related materials. $J$. Appl. Phys. 2013, 113, 231101.

[23] Otto, J.G.; Amani, M. Thermoelectric Properties of $\mathrm{Zn}_{\mathrm{x}} \mathrm{In}_{\mathrm{y}} \mathrm{O}_{\mathrm{x}+1.5 \mathrm{y}}$ Films. J. Electrochem. Soc. 2011, 158, J15-J19.

[24]Lee, H.J.; Hyun, S.; Park, H.S.; Han, S.W. Thermoelectric properties of n-type Bi-Te thin films with various compositions. Microelectronic Engineering 2011, 88, 593-596.

[25]Watanabe, M.; Kita, T.; Fukumura, T.; Ohtomo, A.; Ueno, K.; Kawasaki, M. High-Throughput Screening for Combinatorial Thin-Film Library of Thermoelectric Materials. J. Comb. Chem. 2008, $10,175-178$.

[26]Tseng, S.C.; Chao, W.H.; Yang, P.H.; Chu, H.S.; Hwang, J.D.; Wu, R.J. Enhanced thermoelectric properties of $\left(\mathrm{Bi}_{0.5} \mathrm{Sb}_{1.5} \mathrm{Te}_{3}\right)_{1-x-y}(\mathrm{PbTe})_{x}\left(\mathrm{Zn}_{4} \mathrm{Sb}_{3}\right)_{y}$ by combinatorial screening. Journal of Alloys and Compounds 2014, 588, 658-661.

[27]Wang, S.H.; Cheng, H.M.; Wu, R.J.; Chao, W.H. Structural and thermoelectric properties of HfNiSn half-Heusler thin films. Thin Solid Films 2010, 518, 5901-5904.

[28]Neubrand, A.; Dadda, J.; Müller, E.; Perlt, S.; Höche, T. Spatially Resolved Thermal Conductivity Measurements Using a Thermoreflectance Microprobe. Journal of Electronic Materials 2013, 42, 2165-2171.

[29]Balk, L.J.; Heiderhoff, R.; Phang, J.C.H.; Thomas, C. Characterization of electronic materials and devices by scanning near-field microscopy. Appl. Phys. A. 2007, 87, 442-449.

[30]Altes, A.; Heiderhoff, R.; Balk, L.J. Quantitative dynamic near-field microscopy of thermal conductivity. J. Phys. D Appl. Phys. 2004, 37, 952-963.

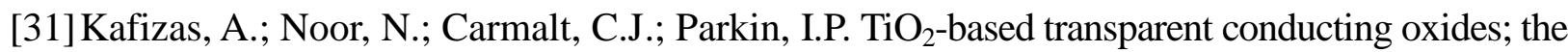
search for optimum electrical conductivity using a combinatorial approach. J. Mater. Chem. C 2013 , $1,6335-6346$.

[32] Shimanovich, K.; Bouhadana, Y.; Keller, D.A.; Rühle, S.; Anderson, A.Y.; Zaban, A. Four-point probe electrical resistivity scanning system for large area conductivity and activation energy mapping. Rev. of Sci. Instrum. 2014, 85, 055103.

[33] Kulikov, V.; Mirsky, V.M. Equipment for combinatorial electrochemical polymerization and high-throughput investigation of electrical properties of the synthesized polymers. Meas. Sci. Technol. 2004, 15, 49-54.

[34]Ramirez, A.G.; Saha, R. Combinatorial studies for determining properties of thin-film goldcobalt alloys. Appl. Phys. Lett. 2004, 85, 5215-5217.

[35]Hewitt, K.C.; Casey, P.A.; Sanderson, R.J.; White, M.A.; Sun, R. High-throughput resistivity apparatus for thin-film combinatorial libraries. R. Rev. Sci. Instrum. 2005, 76, 093906.

[36] Alexander, S.J.; Lin, T.; Brett, D.J.L.; Evans, J.R.G.; Cibin, G.; Dent, A.; Sankar, G.; Darr, J.A. A combinatorial nanoprecursor route for direct solid state chemistry: Discovery and electronic properties of new iron-doped lanthanum nickelates up to $\mathrm{La}_{4} \mathrm{Ni}_{2} \mathrm{FeO}_{10-}$. Solid State Ionics 2012, 225, 176-181.

[37]Polytec GmbH, Datasheet: Model 680 Dynamic Four-Point Probe System, Rev1.2, http://www.polytec.com/fileadmin/user_uploads/Products/PV-und_Halbleiter-Messgeraete/Doc uments/PH_HL_4D_Brochure_680_rev_1_2.pdf, Retrieved: 02. October 2016.

[38]Petersen, C. L.; Hansen, T.M.; Bøggild, P.; Boisen, A.; Hansen, O.; Hassenkam, T.; Grey, F. Scanning microscopic four-point conductivity probes. Sensors and Actuators A 2002, 96, 53-58.

[39] www.capres.com

[40]Thienhaus, S.; Hamann, S.; Ludwig, A. Modular high-throughput test stand for versatile screening of thin-film materials libraries. Sci. Tech. Adv. Mater 2011, 12, 54206. 
[41]Itaka, K.; Minami, H.; Kawaji, H.; Wang, Q.; Nishii, J.; Kawasaki, M.; Koinuma, H. High-speed evaluation of thermoelectric materials using multi-channel measurement system. $J$. of Thermal Analysis and Calorimetry 2002, 69, 1051-1058.

[42] Otani, M.; Thomas, E.L.; Wong-Ng, W.; Schenck, P.K.; Chang, K.S.; Lowhorn, N.D.; Green, M.L.; Ohguchi, H. A High-Throughput Screening System for Thermoelectric Material Exploration Based on a Combinatorial Film Approach. Japanese Journal of Applied Physics 2009, 48, 05EB02.

[43]Funahashi, R.; Mikami, M.; Urata, S.; Kitawaki, M.; Kouuchi, T.; Mizuno, K. High-throughput screening of thermoelectric oxides and power generation modules consisting of oxide unicouples. Meas. Sci. Technol. 2005, 16, 70.

[44]Datasheet penta 0 probes from manufacturer IDI. http://www.scp-sa.es/resources/upload/files/categorias/329/esp/IDI_Interconnect_37_101.pdf

[45] Yamamoto, A.; Michihiro, O.; Lee, C.H.; Masayuki, M. High throughput screening of thermoelectric materials - preparation and measurement of ternary thin film library. Presentation on the $34^{\text {th }}$ ICT and $13^{\text {th }}$ ECT 2015, Dresden, Gemany.

[46] Yamamoto, A.; Noguchi, T.; Obara, H.; Ueno, K.; Ikeuchi, S.; Sugawara, T.; Shimada, K.; Takasaki, Y.; Ishii, Y. Combinatorial Approach for Thermoelectric Materials through Bulk Composition-Spreads and Diffusion Multiples. Mater. Res. Soc. Symp. Proc. 2008, 1024, A01-05.

[47] Yamamoto, A.; Kukuruznyak, D.; Ahmet, P.; Chikyow, T.; Ohuchi, F.S. High-Throughput Screening of Thermoelectric Materials; Application of Thermal Probe Method to Composition-Spread Samples. Mat. Res. Soc. Symp. Proc. 2004, 804, JJ1.4.

[48]Iwanaga, S.; Toberer, E.S.; LaLonde, A.; Snyder, G. J. A high temperature apparatus for measurement of the Seebeck coefficient. Rev. Sci. Instrum. 2011, 82, 063905.

[49]de Boor, J.; Stiewe, C.; Ziolkowski, P.; Dasgupta, T.; Karpinski, G.; Lenz, E.; Edler, F.; Mueller, E. High temperature measurement of Seebeck coefficient and electrical conductivity. Journal of Electronic Materials 2013, 42, 1711-1718.

[50] Yamamoto, A. ; Obara, H.; Ueno, K. Optimization of Thermoelectric Properties of Ni-Cu based Alloy through Combinatorial Approach. Mater. Res. Soc. Symp. Proc. 2008, 1044, U06-18.

[51]Ziolkowski, P. ; Karpinski, G.; Dasgupta, T.; Müller, E. Probing thermopower on the microscale. Physica Status Solidi a - Applications and Materials Science 2013, 210, 89-105.

[52]Wu, K.-H.; Hung, C.-I.; Ziolkowski, P.; Platzek, D.; Karpinski, G.; Stiewe, C.; Müller, E. Improvement of spatial resolution for local Seebeck coefficient measurements by deconvolution algorithm. Rev. Sci. Instrum. 2009, 80, 105104.

[53]Ziolkowski, P.; Karpinski, G.; Platzek, D.; Stiewe, C.; Müller, E. Application Overview of the Potential Seebeck Microscope. Proc. 25th ICT 2006, 684-688.

[54]Jung, D.; Kurosaki, K.; Kim, C.; Muta, H.; Yamanake, S. Thermal expansion and melting temperature of the half-Heusler compounds: MNiSn (M = Ti, Zr, Hf). Journal of Alloys and Compounds 2010, 489, 328-331.

[55]Rogl, G.; Grytsiv, A.; Gürth, M.; Tavassoli, A.; Ebner, C.; Wünschek, A.; Puchegger, S.; Soprunyuk, V.; Schranz, W.; Bauer, E.; Müller, H.; Zehetbauer, M.; Rogl, P. Mechanical properties of half-Heusler alloys. Acta Materialia 2016, 107, p. 178-195.

[56]Maseeh, F.; Gelston, S.M.; Senturia, S.D. Mechanical Properties of Microelectronic Thin Films: Silicon DiOxide $\left(\mathrm{SiO}_{2}\right)$. VLSI Publications 1989, 89, 575.

[57] Okada, Y.; Tokumaru, Y. Precise determination of lattice parameter and thermal expansion coefficient of silicon between 300 and 1500 K. Journal of Applied Physics 1984, 56, 314.

[58] Yim, W.M.; Paff, R.J. Thermal expansion of AlN, sapphire, and silicon. Journal of Applied Physics 1974, 45, 1456. 
[59] Martienssen, W.; Warlimont, H. Springer handbook of condensed matter and materials data. Springer 2005, 445-446, p. 438-439.

[60]Figge, S.; Kröncle, H.; Hommel, D.; Epelbaum, B.M. Temperature dependence of the thermal expansion of AlN. Appl. Phys. Lett. 2009, 94, 101915.

[61] Gang Zhang, G.; Zhang, Y.W. Strain effects on thermoelectric properties of two-dimensional materials. Mechanics of Materials 2015, 91, p. 382-398.

[62] Cuong, D.D.; Rhim, S.H.; Lee, J.-H.; Hong, S.C. Strain effect on electronic structure and thermoelectric properties of orthorhombic SnSe: A first principles study. AIP Advances 2015, 5, 117147.

[63]Hinsche, N.F.; Mertig, I.; Zahn, P. Effect of strain on the thermoelectric properties of silicon: an ab initio study. J. Phys.: Condens. Matter 2011, 23, 295502.

[64]Pei, Q.-X.; Zhang, Y.-W.; Sha, Z.-D.; Shenoy, V. B. Tuning the thermal conductivity of silicene with tensile strain and isotopic doping: A molecular dynamics study, J. Appl. Phys. 2013, 114, 033526.

[65]Lv, H. Y.; Lu, W. J.; Shao, D. F.; Lu, H. Y.; Sun, Y. P. Strain-induced enhancement in the thermoelectric performance of a $\mathrm{ZrS}_{2}$ monolayer. J. Mater. Chem. C 2016, 4, 4538.

[66]Guo, S-D.; Wang, Y. Small compressive strain induced semiconductor-metal transition and tensile strain enhanced thermoelectric properties in monolayer $\mathrm{PtTe}_{2}$. Semiconductor Science and Technology 2017, 32, 055004.

[67]Jaeger, T.; Mix, C.; Schwall, M.; Kozina, X.; Barth, J.; Balke, B.; Finsterbusch, M.; Idzerda, Y. U.; Felser, C.; Jakob, G. Epitaxial growth and thermoelectric properties of TiNiSn and $\mathrm{Zr}_{0.5} \mathrm{Hf}_{0.5} \mathrm{NiSn}$ thin films. Thin Solid Films 2011, 520, 1010.

[68] Glassbrenner, C. J.; Slack, G. A. Thermal Conductivity of Silicon and Germanium from $3^{\circ} \mathrm{K}$ to the Melting Point. Phys. Rev. 1964, 134, A1058.

[69]Burghartz, St.; Schulz, B. Thermophysical properties of sapphire, $\mathrm{AlN}$ and $\mathrm{MgAl}_{2} \mathrm{O}_{4}$ down to 70 K. J. Nuclear Materials 1994, v212-215, p1065.

[70]Datasheet of AlN of the Accuratus Ceramic Corporation: http://accuratus.com/alumni.html

[71]Colosky, B.P. Thermal conductivity measurements on silica. American Ceramic Soc. Bull. 1952, v31(1), p465.

[72]Zhou, A. ; Wang, W.; Yao, X.; Yang, B.; Li, J.; Zhao, Q.; Wang, C.; Xu, D.; Ziolkowski, P.; Müller, E. Impact of the film thickness and substrate on the thermopower measurement of thermoelectric films by the potential-Seebeck microprobe (PSM). Applied Thermal Engineering 2016, 107, 552-559.

[73]Jaeger, T.; Mix, C.; Schwall, M.; Kozina, X.; Barth, J.; Balke, B.; Finsterbusch, M. Idzerda, Y.U.; Felser, C.; Jakob, G. Epitaxial growth and thermoelectric properties of TiNiSn and $\mathrm{Zr}_{0.5} \mathrm{Hf}_{0.5} \mathrm{NiSn}$ thin films. Thin Solid Films 2011, 520, 1010-1014.

[74] Kozina, X,; Jaeger, T.; Ouardi, S.; Gloskowski, A.; Stryganyuk, G.; Jakob, G.; Sugiyama, T.; Ikenaga, E.; Fecher, G.H.; Felser, C. Electronic structure and symmetry of valence states of epitaxial NiTiSn and $\mathrm{NiZr}_{0.5} \mathrm{Hf}_{0.5} \mathrm{Sn}$ thin films by hard $\mathrm{x}$-ray photoelectron spectroscopy. Applied Physics Letters 2011, 99, 221908. 